\title{
La raza bovina autóctona española Pajuna: Situación actual y programa de recuperación ${ }^{\mathrm{a}}$
}

\author{
A. Luque 1 , M. Valera ${ }^{2}$, P.J. Azor ${ }^{3}$, F. Goyache ${ }^{4}$, E. Rodero ${ }^{5} \&$ A. Molina ${ }^{3}$ \\ ${ }^{1}$ Asociación de Criadores de Ganado Vacuno de Raza Pajuna (GRAPA), \\ Partido Rural de Sigüela s/n, Apto. Correos 159, 29400 Ronda, Málaga, España \\ ${ }^{2}$ Departamento de Ciencias Agroforestales, EUITA, Universidad de Sevilla, \\ Crtra. de Utrera Km. 1, 41013 Sevilla, España \\ ${ }^{3}$ Departamento de Genética, Universidad de Córdoba, Campus Universitario de Rabanales, Edificio \\ Mendel, Crtra. Madrid-Cádiz, km. 373a, 14071 Córdoba, España \\ ${ }^{4}$ Área de Genética y Reproducción Animal, Serida-Somió, 33203 Gijón, España \\ ${ }^{5}$ Departamento de Producción Animal, Universidad de Córdoba, Campus Universitario de \\ Rabanales, Crtra. Madrid-Córdoba, km. 373a., 14071 Córdoba, España
}

\section{Resumen}

La raza bovina Pajuna es una raza autóctona andaluza que está al borde de la extinción. Su aptitud es mixta carne-trabajo y es posiblemente la raza española más rústica, al ser capaz de adaptarse a los medios marginados, aprovechando los escasos recursos de las sierras andaluzas, que no pueden ser utilizados por otras razas más carniceras.

El cruzamiento indiscriminado con razas especializadas, la mecanización del campo y la pérdida del hábitat de explotación han llevado esta raza a una situación de inminente desaparición, con sólo 300 animales puros, relegados en la mayoría de los casos a meros vientres para el cruce industrial en vacadas mixtas.

En el presente artículo describimos la situación actual y las causas que la han llevado a esta situación, así como las actuaciones de la Asociación de Criadores (GRAPA), creada en el año 2001, dentro de un programa de preservación y recuperación. Estas razones se pueden resumir en la localización de todos los efectivos existentes, el análisis de la estructura demográfica de la población, la actualización de su estándar racial y creación del Libro Genealógico, la caracterización genética, y determinación del nivel de variabilidad intra e interpoblacional a partir del polimorfismo de loci moleculares microsatélites como a través de la variabilidad de la región control del ADN mitocondrial.

\section{Summary}

The Pajuna is a cattle breed native to Andalusia in Spain. It is an endangered breed exhibiting both meat quality and draft animal attributes. The Pajuna breed is possibly the most resilient bovine breed $\mathrm{s}$ in Spain. This breed is adapted to adverse conditions, taking advantage of the limited resources of the Andalusian mountains which cannot be used by other breeds. However, massive crossbreeding with other breeds, the mechanization of rural areas and the loss of its habitat have led this breed to the brink of extinction. At the moment there are 300 pure animals, most of which are females destined for industrial crossing in

\footnotetext{
${ }^{a}$ Este trabajo ha sido financiado gracias al proyecto IN IA RZ 02-007. Caracterización socioeconómica, productiva y genética de la raza bovina Pajuna: Bases para el desarrollo de estrategias para su preservación, conservación y desarrollo sostenible
} 
mixed farms. The present work is intended to describe the causes of this situation. The Association of Breeders (GRAPA), created in 2001, is working towards a program of preservation and recovery for this breed and accordingly the following steps have been taken. The existing population of Pajunas has been identified, and an analysis made of its demographic structure allowing for the establishment of a breed standard and the creation of a studbook. We have also genetically characterized this breed. The level of inter- and intra-population variability has been determined using polymorphism of microsatellites loci and D-loop region of the mitochondrial DNA (mtDNA).

Palabras clave: Peligro de extinción, Razas bovinas, Libro Genealógico, Variabilidad genetica, Nivel de pureza, Reacciones de PCR múltiple, Diversidad genotípica, Coeficiente de consanguinidad.

\section{In troducción}

La raza bovina Pajuna, es una de las razas bovinas andaluzas consideradas en inminente peligro de extinción debido principalmente al cruzamiento con otras razas selectas (Molina et al., 2005). El RD 1682/ 1997, de 7 de noviembre, en el que se actualiza el catálogo oficial de razas de ganado de España, la considera como una raza de protección especial. También se le conoce por Serrana, debido a ser considerada como la raza de las sierras andaluzas, y como Castellana.

Por su gran rusticidad, es capaz de adaptarse a los medios más difíciles (en terrenos escarpados y fríos de alta montaña, a veces nevados y frecuentemente compartiendo pastos con rebaños caprinos y/ o cinegéticos), aprovechando los escasos recursos de los medios marginales, siendo explotada de forma extensiva para carne, contribuye por un lado a la conservación de estas zonas y por otro al mantenimiento de la población humana, siendo un claro ejemplo de desarrollo sostenible (Luque et al., 2001).
Es conocida por la calidad de su carne roja, aunque los antiguos pastencos, que son canales de becerros de seis a ocho meses, criados a hierba y $140 \mathrm{~kg}$. de peso vivo, ya no son comerciales y son vendidos a cebaderos (Luque et. al., 2003).

Tradicionalmente era explotada por su aptitud trabajo en las zonas costeras de Málaga a Almería y en la Sierra de la Axarquía (Málaga), donde aun quedan gañanes, siendo pieza clave de sus economías rurales en las que era explotada para labrar sus huertas, alimentándose de los rastrojos, estercolando las tierras de labor y dando a su vez un becerro, pero debido a la mecanización del campo, actualmente solo se enganchan toros para romerías. Se surtían de ganaderos que pasaban desde otoño hasta final de la primavera en Sierra Morena, Sierras de Cazorla, Segura y Villas y Serranía de Ronda, Alcornocales, Grazalema e incluso el valle de Alcudia en Castilla la Mancha (donde se apreciaba su aptitud trabajo en las minas), y trashumaban en el estío hacia las Alpujarras y Sierra Nevada. En la actualidad no se llevan a cabo estas prácticas de manejo (Figura 1).

\section{Material y Métodos}

Se han localizado todas las explotaciones que poseían algún animal de raza Pajuna y se ha abierto el Libro Genealógico de la raza, incluyendo a los animales en los diferentes registros en función del nivel de pureza. Para ello ha sido necesario discriminar entre los animales que se pueden considerar etnozootécnicamente puros de los animales que presenten una mayor o menor influencia de otras razas, utilizando el patrón de la raza propuesto por Herrera et al. (1996).

La estimación del tamaño efectivo de la población $(\mathrm{Ne})$ se obtuvo siguiendo las fórmulas de Latter-Hill y de Wright. El modelo de Latter-Hill (Latter, 1959; Hill, 1972, 1979) viene representado por la siguiente ecuación: 


$$
\begin{aligned}
& \frac{1}{\mathrm{~N}_{\mathrm{e}}}=\frac{1}{16 \mathrm{FL}}\left[2+\sigma^{2}\left(\mathrm{X}_{\mathrm{mm}}\right)+2(\mathrm{M} / \mathrm{F}) \sigma\left(\mathrm{X}_{\mathrm{mm}}, \mathrm{X}_{\mathrm{nf}}\right)+(\mathrm{M} / \mathrm{F})^{2} \sigma^{2}\left(\mathrm{X}_{\mathrm{nf}}\right)\right] \\
& +\frac{1}{16 \mathrm{FL}}\left[2+(\mathrm{F} / \mathrm{M})^{2} \sigma^{2}\left(\mathrm{X}_{\mathrm{fin}}\right)+2(\mathrm{~F} / \mathrm{M}) \sigma\left(\mathrm{X}_{\mathrm{ffm}}, \mathrm{X}_{\mathrm{ff}}\right)+\sigma^{2}\left(\mathrm{X}_{\mathrm{ff}}\right)\right]
\end{aligned}
$$

donde $\mathrm{M}$ y $\mathrm{F}$ representan el número de machos y de hembras en edad reproductiva por año; L es el intervalo medio entre generaciones, expresado en años; Xij es el tiempo de vida de la progenie de sexo j del padre de sexo i.

Esta ecuación tiene en cuenta la distribución observada del tamaño de progenie. Sin embargo si asumimos que todos los individuos tienen la misma posibilidad, durante una unidad de tiempo dada, de contribuir a la siguiente generación, la distribución del tamaño de progenie se aproxima a una distribución de Poisson (Kimura and Crow, 1963) qued ando la ecuación de Ne como la de Wright (1931): $\mathrm{Ne}=(4 \mathrm{MLFL}) /(\mathrm{ML}+\mathrm{FL}) . \mathrm{El}$ incremento de consanguinidad por generación puede aproximarse a $1 /(2 \mathrm{Ne})$.

Se ha realizado la valoración etnozootécnica a una prospección de 38 ganaderías y 618 animales (584 hembras y 34 sementales), lo que representa la mitad de las hembras reproductoras y todos los sementales funcionales en la actualidad. Esto permitió la inclusión en el libro genealógico de 22 machos y 295 hembras puras. Se realizaron 6 medidas zoométricas a 71 animales adultos ( 8 machos y 63 hembras). La caracterización morfológica y faneróptica fue llevada a cabo en 352 animales siguiendo la metodología propuesta por Jordana y Ribo (1991) y Rodero et al., (1994), que incluye 30 variables cualitativas.

Para la caracterización genética de la raza Pajuna, el análisis de la variabilidad intra e interpoblacional y la elección del panel de marcadores más eficaz para el control de filiación en esta raza, se han genotipado 51 animales, utilizado 31 microsatélites recomendados por el grupo de expertos de la FAO (1999) e ISAG para estudios de diversidad genética en bovino. Además de los 51 animales pajunos analizados, en determinados análisis se han utilizado las frecuencias alélicas de 25 animales de raza Berrenda en Negro, 25 de Berrenda en Colorado y 14 animales de raza Cárdena And aluza (Rodero, 2003).

El procesado de las muestras se ha llevado a cabo según técnicas estándares puestas a punto en nuestro laboratorio de genética molecular. Para su amplificación se han agrupado en reacciones de PCR múltiple (Azor et al., 2003). Las frecuencias alélicas se han calculado mediante recuento directo.

La variabilidad genética que se mantiene en la población actual se ha estimado mediante diferentes parámetros (diversidad alélica, heterocigosidad observada y esperada, el número efectivo de alelos y la diversidad genotípica). Para conocer la estructura genética de las subpoblaciones de la raza Pajuna, hemos estimado los estadísticos-F de Wright $\left(\mathrm{F}_{\mathrm{IS}}, \mathrm{F}_{\mathrm{IT}}\right.$ y $\left.\mathrm{F}_{\mathrm{ST}}\right)$. Así mismo hemos estimado la distancia genética de Reynolds (Reynolds et al., 1983), una distancia que se ha mostrando muy adecuada para la diferenciación de poblaciones que han divergido hace muy poco tiempo. Finalmente se ha realizado un análisis de la posibilidad de asignar los distintos individuos analizados (genotipos) a sus respectivas poblaciones. Para ello se ha utilizado el procedimiento de máxima verosimilitud con remuestreo bootstrapping (Paetkau et al. 1995, 1997).

El coeficiente de consanguinidad ha sido estimado de forma indirecta a partir de las heterocigosidades observadas y esperadas del grupo de marcadores genéticos siguiendo la metodología propuesta por Nei y Kumar (2000).

Para la estimación del parentesco molecular entre los diferentes individuos, se ha utilizado el estimador R (total relatedness estimate) definido por Wang (2002), a partir 


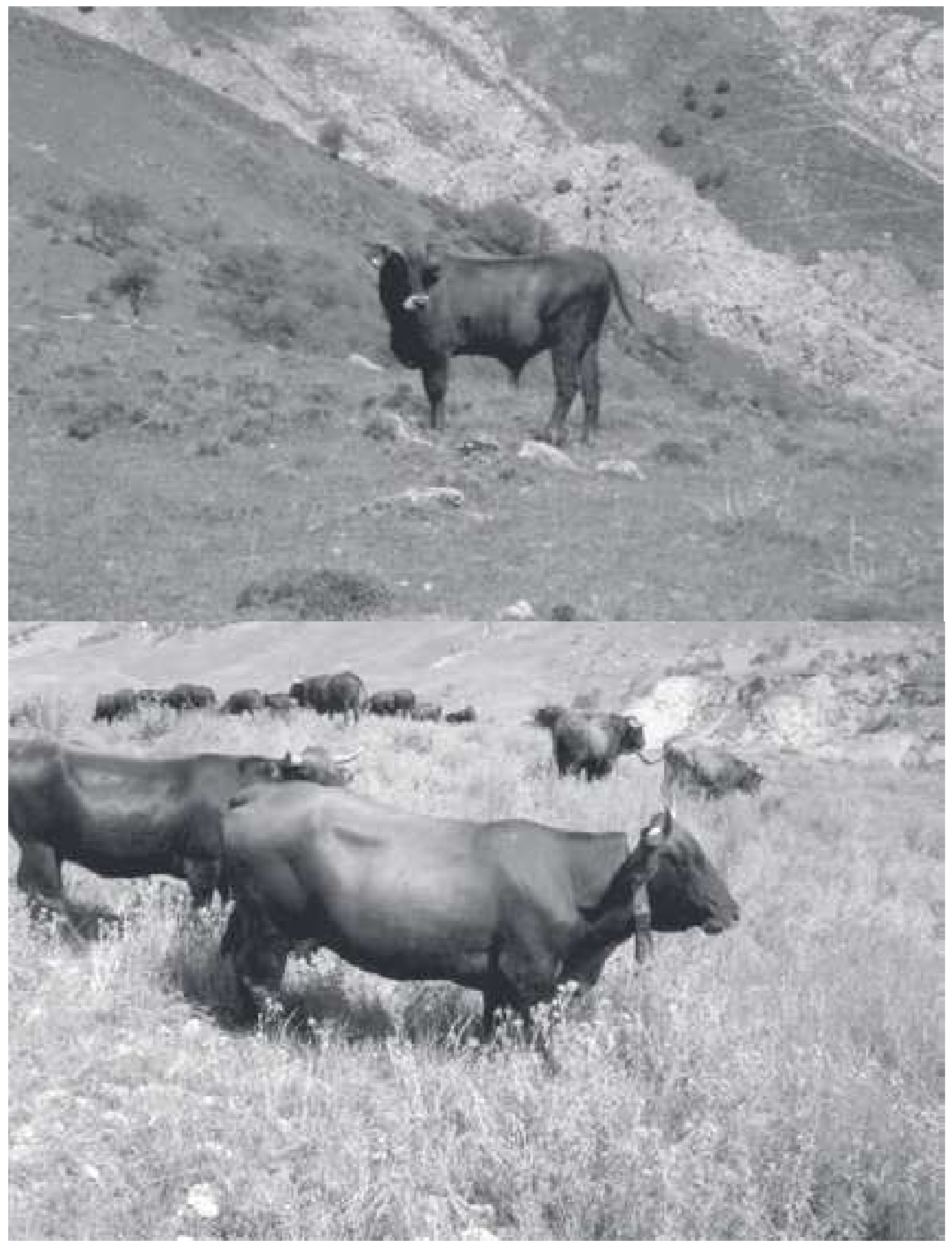

Figura 1. Rebaño de vacas de raza Pajuna en su medio ambiente típico. 
de los estimadores PHI y DELTA (Lynch and Ritland, 1999, Li et al., 1993).

Con respecto al análisis del $\mathrm{ADN}$ mitocondrial en la raza Pajuna, se ha realizado la amplificación de la región D-loop utilizando los oligonucleótidos y el protocolo publicados por Loftus et al. (1994) para su posterior secuenciación en 9 individuos de raza Pajuna seleccionados para encontrar la mayor diversidad posible de orígenes. Para la amplificación de esta región del ADN mitocondrial se ha seguido la metodología expuesta por Cymbron et al. (1999), mediante PCR con oligonucleótidos diseñados por estos autores, denominados AN4 (marcado) y AN3 permiten la amplificación de un fragmento de 375 pares de bases de la región de control del ADN mitocondrial bovino. Asimismo se han obtenido de GenBank las correspondientes secuencias de 10 individuos de raza Limousin (números de acceso AF336502-11), 12 de raza Charolais (números de acceso AF336523-33 y U51817), 11 de raza Brown Swiss (números de acceso AB079362-4, AF16072-78 y AF034438), 2 de raza Retinta (números de acceso AF531410-11), y 11 de raza N'Dama (números de acceso AF336656-63 y U51837-9). Con ello se pretende conocer el grado de influencia del ganado africano más representativo (N'Dama), y alguna de las razas europeas genotipadas que más presencia han tenido en tiempos recientes en el área geográfica en la población Pajuna.

\section{Resultados y Discusión}

\section{Evolución censal y situación actual}

Según la escasa bibliografía disponible, sus censos han disminuido de forma vertiginosa en los últimos años, bajando desde un par de miles de hembras de raza pura (Aparicio, 1960; Sánchez-Belda, 1984) a varios cientos (Rodero et al., 1992). Los resultados obtenidos han permitido estimar un censo total de la raza de 1367 cabezas de ganado vacuno con base genética de raza Pajuna, con distintos niveles de pureza, distribuidos en 106 ganaderías de 9 provincias (todas las andaluzas y Ciudad Real), y un tamaño medio de 12 cabezas/ explotación. Estos censos son aparentemente muy superiores a los recogidos en la bibliografía mas reciente (Rodero 1999; Sánchez-Belda, 2002), o a las 310 hembras reproductoras con once machos que estiman las bases de datos EAAP y FAO, si bien hay que tener en cuenta que estos últimos sólo incluían animales puros en ganaderías con censos reseñables.

Estas 106 explotaciones pueden ser clasificadas en:

熙 19 ganaderías con algún semental de raza Pajuna (cría en pureza aunque también pueda realizar cruce industrial) y un censo total de 416 animales.

颐 27 ganaderías dedicadas al cruce industrial (exclusivamente sementales de razas cárnicas especializadas y madres con fuerte base genética Pajuna) y un censo de 719 animales.

㯺53 ganaderías de otras razas que cuentan en su cabaña con alguna cabeza de ganado pajuno de distinta procedencia (208 animales pajunos).

涌 7 Domadores o gañanes, con un censo de 24 animales que no se aparean (adultos no reproductores).

Destaca que sólo 19 ganaderos poseen al menos un semental de raza Pajuna (menos del 18\% del total), cuentan con 333 hembras reproductoras ( $36 \%$ del total de la raza), de las que 121 son del nivel A ( $38 \%$ del total de este nivel y $10,7 \%$ del total de la raza).

Debido a que la reposición es realizada con novillas de raza Pajuna, el nivel de hembras puras en este núcleo es el más numeroso, superior a la media de toda la raza que es del $25 \%$.

Sólo dos ganaderías poseen más de cincuenta cabezas. Este hecho hace que los ingresos de esta explotación tengan que complementarse con otras actividades, lo que implica que hoy día la explotación del vacuno Pajuno sea una actividad a tiempo parcial poco especializada. Del censo total de animales reproductores de raza Pajuna con un nivel aceptable de pureza (1 133 hembras 
reproductoras y 34 toros), tan sólo 287 vacas de vientre y 22 sementales se encuadran en el nivel A (animales puros).

En cuanto a la distribución, según nuestros resultados, a pesar de que su hábitat tradicional ha sido muy amplio, prácticamente todas las serranías y sierras andaluzas, en la actualidad está restringida a núcleos muy reducidos en la Sierra Norte de Sevilla y Sierra Morena Cordobesa, en las Alpujarras Granadinas (animales todos cruzados), Sierras de Cazorla, Segura y Villas e inmediaciones, y animales en vacadas mixtas de la Serranía de Ronda y Sierra de Grazalema (Figura 2). También hemos detectado reductos en la Axarquía malagueña y un rebaño en Almadén (Ciudad Real).

Según su localización geográfica y flujo de genes hemos distinguido cinco zonas de influencia claramente diferenciadas. Éstos en la práctica se están comportando hoy día como núcleos aislados, sin flujo genético entre ellos:
1. Núcleo de Jaén (La Carolina y Sierras de Cazorla, Segura y las Villas).

2. Núcleo de Ronda (Sierra de Grazalema, Parque de los Alcornocales y Serranía de Ronda). También llamado núcleo de Málaga.

3. Núcleo de Granada (Cara Norte de Sierra Nevada, Güejar-Sierra, Alpujarras y Almería).

Los núcleos Jaén, Ronda y Granada coinciden con tres áreas geográficas más o menos bien delimitadas y separadas entre ellas por zonas no ganaderas de campiña.

4. Equidistante entre las tres, en la Sierra de la Axarquía, se sitúa un cuarto núcleo, donde la cría de ganado es casi testimonial y es la única que aún selecciona para aptitud trabajo (Enganche o gañanes).

5. Por último, localizamos un núcleo disperso que denominamos Otros, muy heterogéneo en cuanto a localización geográfica y sistemas de explotación, formado por animales sueltos insertos en

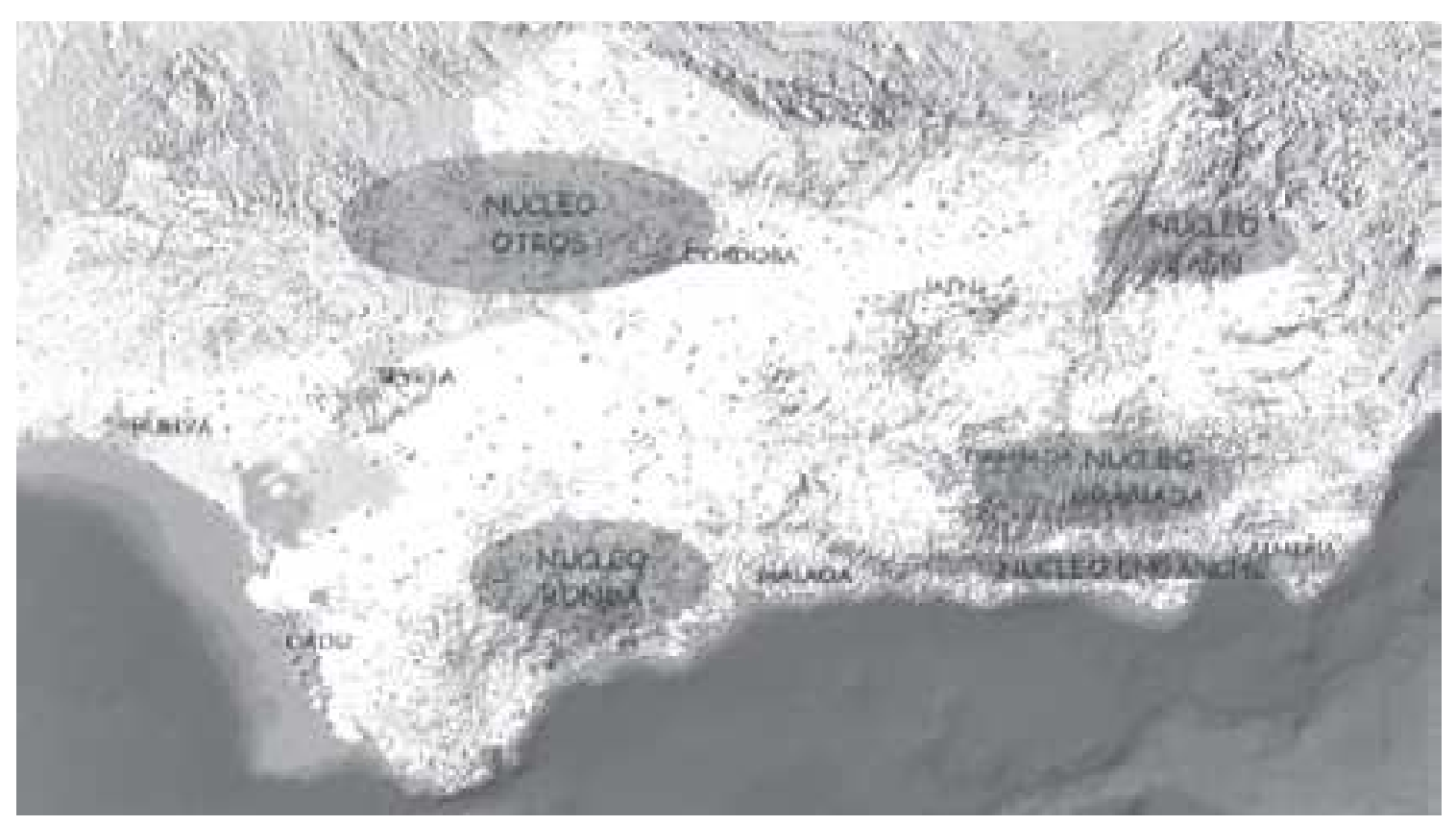

Figura 2. Localización de los principales núcleos geográficos de ganaderías de raza Pajuna, en el sur de España. 
vacadas mixtas, que por distintos motivos han terminado fuera de su ubicación habitual.

En la tabla 2 se presenta el tamaño efectivo de cada Núcleo utilizando los censos de la tabla 1 y asumiendo que no existe flujo genético entre los Núcleos y tampoco restricciones al movimiento dentro de cada uno de ellos. Sería por tanto la situación equivalente a la existente en poblaciones pequeñas mantenidas para la preservación en pequeños núcleos aislados.

El tamaño efectivo determina los niveles de deriva genética y consanguinidad y por tanto la pérdida de variabilidad en las pequeñas poblaciones. Es de mucha importancia la posibilidad o no de flujo de reproductores entre núcleos y ganaderías. Así, el concepto de tamaño efectivo de la población ( $\mathrm{Ne}$ ) es importante para comprender los efectos de la variación en el número de machos y de hembras y del tamaño de familia sobre la deriva genética y la consanguinidad. En función de este Ne la raza Pajuna se encuentra en "peligro sostenido".

Estos resultados son equiparables a los estimados por la EAAP $(\mathrm{Ne}=16)$, en donde se estima un incremento por generación del $3,13 \%$ de consanguindad.

Se denota que un bajo número de machos reduce el tamaño efectivo de la población drásticamente. Así mismo se puede observar como de seguir el actual aislamiento entre núcleos la situación sería irreparable en muy pocas generaciones.

Las causas por las que ha llegado a la situación en que se encuentra, son básicamente tres (Molina et al., 2003): El cruce indiscriminado con otras razas

Tabla 1. Distribución estimada del número de animales reproductores por núcleo y nivel de pureza.

\begin{tabular}{lrrrrrrrr}
\hline & \multicolumn{3}{c}{ Machos } & \multicolumn{7}{c}{ Hembras } & \\
\cline { 2 - 7 } Zona/ Nivel de pureza & \multicolumn{1}{c}{ A } & B & Total & \multicolumn{1}{c}{ A } & B & C & Total & Ganaderos \\
\hline Jaén & 3 & 1 & 4 & 71 & 54 & 37 & 162 & 17 \\
Ronda & 5 & 0 & 5 & 50 & 43 & 28 & 121 & 24 \\
Granada & 6 & 3 & 9 & 105 & 272 & 365 & 742 & 38 \\
Otros & 2 & 0 & 2 & 41 & 16 & 3 & 60 & 14 \\
Axarquía & 6 & 8 & 14 & 20 & 17 & 11 & 48 & 13 \\
Total & 22 & 12 & 34 & 287 & 402 & 444 & 1133 & 106 \\
\hline
\end{tabular}

Tabla 2. Número efectivo e incremento de consanguinidad esperada en la población de animales Pajunos considerando cuatro nú cleos aislados.

\begin{tabular}{clcccc}
\hline & & Jaén & Granada & Málaga & Otros \\
\hline \multirow{2}{*}{$\mathrm{N}^{\circ}$ machos } & A: 15 & 3 & 5 & 5 & 2 \\
& B: 1 & 0 & 1 & 0 & 0 \\
& A: 110 & 47 & 21 & 29 & 13 \\
$\mathrm{~N}^{\circ}$ hembras & $\mathrm{B}: 99$ & 35 & 36 & 35 & 3 \\
& $\mathrm{C}: 65$ & 15 & 34 & 15 & 1 \\
$\mathrm{~N}$ total & & 100 & 97 & 84 & 19 \\
$\mathrm{Nm}$ & 3 & 6 & 5 & 2 \\
$\mathrm{Nh}$ & & 74,5 & 22,2 & 71,5 & 16,5 \\
$\mathrm{Ne}$ & & 11,6 & 18,7 & 7,1 \\
$\Delta \mathrm{F}$ & & $4,3 \%$ & $2,3 \%$ & 2,7 & $7,0 \%$ \\
\hline
\end{tabular}

Nm: Número de machos; Nh: número de hembras; Ne: Tamaño efectivo; $\Delta F$ : Incremento de consanguinidad por generación. 
foráneas (que ha hecho que en algunas explotaciones con vocación cruce industrial, no quede ninguna vaca clasificada en A), falta de criterios de selección o criterios obsoletos (aptitud trabajo aunque puede tener cierta demanda para tirar de las carretas en las romerías) y el desplazamiento de la actividad ganadera en estas zonas por otras más cómodas o rentables (ganado caprino o cinegético y turismo rural principalmente).

\section{Caracterización morfológica de la raza Pajuna}

Según nuestros resultados la raza Pajuna es ortoide, mediolínea, de piel basta, de capa castaña con oscurecimientos centrífugos y particularmente bociclara, capa de toros más oscura que hembras y abundante pelo rojo encendido en testuz, mucosas oscuras, de subhipermétrica a subelipométrica, con pesos medios de $375 \mathrm{~kg}$ las hembras a $600 \mathrm{~kg}$ los machos, variando mucho según la alimentación recibida, según dos modas: Ganado de piara o domado; de esta forma, encontramos ejemplares aislados insertos en vacadas mixtas en pastos de buena calidad, o criados en pesebre y domados para tiro, que alcanzan $550 \mathrm{~kg}$. las hembras y $1000 \mathrm{~kg}$. los machos. Se corresponden con el morfotipo ambiental (Aparicio, 1960), así, las extremidades son largas para poder defenderse en las zonas escarpadas donde suele pastar, aunque en los animales seleccionados para tiro, el índice de compacidad aumenta, cabeza voluminosa, y cuerna de sección circular en gancho abierto, blancos por la base y negros o caobas por la punta, escaso diámetro bicostal, cruz destacada, ensillado frecuente y de escaso desarrollo muscular, grupa de longitud media, cerrada posteriormente y tendente a la horizontalidad (Figura 3).

El análisis estadístico determinó unos elevados coeficientes de variación y unas significativas diferencias entre Núcleos para la mayoría de las variables zoométricas. En cuanto a los caracteres morfológicos y fan erópticos, se detectaron diferencias significativas en 14 de las 30 variables analizadas. Estas diferencias son reflejo por una parte del aislamiento reproductivo entre las ganaderías de cada Núcleo y por otra a una selección de la reposición buscando un tipo de animal diferente (y probablemente también a diferente influencia de otras razas en el pasado). El análisis clúster determinó un mayor agrupamiento entre los Núcleos de Jaén y Málaga y una mayor diferenciación en el de Otros. Así mismo el porcentaje de agrupamiento correcto en el análisis discriminante fue del $71 \%$, siendo el Núcleo de Granada con un $82 \%$ el que presenta una mejor definición, y el de Ronda (52\%) el peor.

Las diferencias significativas observadas son indicativas de unas mayores diferencias fanerópticas que zoométricas, y que éstas se acusan más entre núcleos que entre sexos, y entre animales seleccionados para aptitud carne o trabajo. Este resultado coincide con Aparicio (1960), Sánchez-Belda (1984) y el propio estándar de la raza que distingue dos zonas de producción primaria y secundaria, o de piara y domado respectivamente.

\section{Caracterización genética de la raza Pajuna}

\section{Estimación de la variabilidad genética poblacional a partir del polimor fismo de los microsatélites del ADN}

Todos los microsatélites utilizados han sido polimórficos. Se han encontrado en esta raza 220 alelos en el total de los marcadores amplificados, siendo el número medio de alelos encontrados por locus de 7,1. Se ha determinado el número de alelos específicos de cada núcleo, es decir, los alelos que se han encontrado en animales de un núcleo que no se han visto en los demás. Se ha visto que hay grandes diferencias en este número de alelos (Malaga=7, Granada=20, Jaén=47). Puesto que las diferencias encontradas pueden estar condicionadas por el tamaño muestral, se hace necesario corregir 

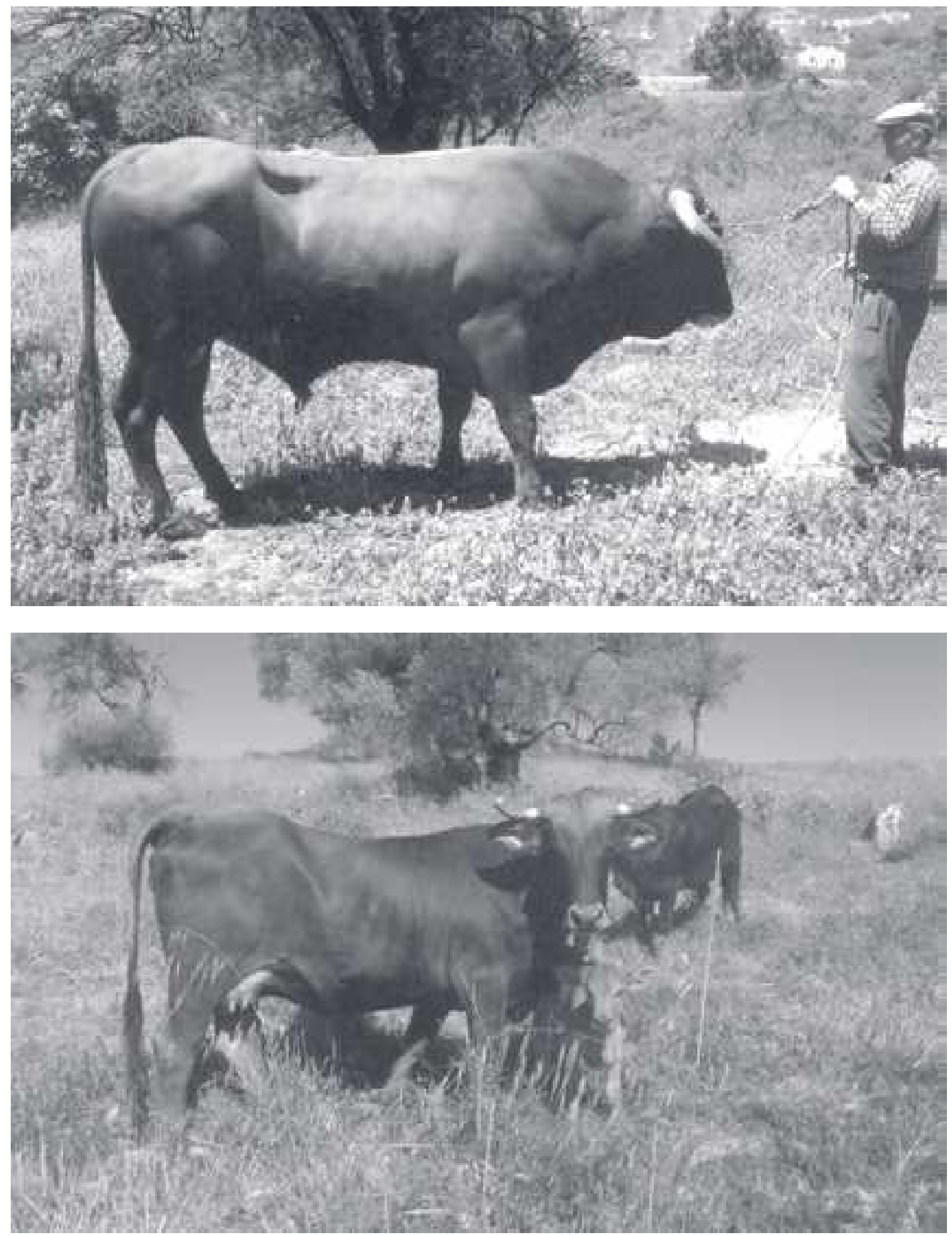

Figura 3. Macho y hembra típicos de la raza Pajuna. 
calculando para ello la riqueza alélica de acuerdo con la corrección de El Mousadik y Petit (1996). En este caso los valores de riqueza alélica en los tres núcleos han sido prácticamente similares (4 alelos).

Los valores de diversidad genética dentro de núcleos $\left(\mathrm{H}_{\mathrm{s}}\right)$ han sido ligeramente inferiores en la mayoría de los loci y en el total de todos ellos $(0,703)$ que los valores obtenidos de diversidad genética total $\left(\mathrm{H}_{\mathrm{T}}=0,716\right)$, lo que indica una pérdida de heterocigotos en las subpoblaciones con respecto a la población en conjunto. En cambio, el valor de $\mathrm{D}_{\mathrm{ST}}$ (diversidad génica entre los núcleos) obtenido para el total de los loci no ha sido muy elevado $(1,2 \%)$, mostrando un escaso peso en las diferencias entre núcleos en relación con las diferencias entre individuos.

En la población global se observa un mayor número efectivo de alelos $(\mathrm{Nea}=3,7)$ (Selander, 1976) con respecto a las subpoblaciones $(3,13$ a 3,5$)$ lo que informa de una pérdida de variabilidad como consecuencia de la subdividisión de una población en pequeños núcleos (efecto Wahlund). En cuanto a la diversidad genotípica (Stoddart y Taylor, 1985) se ha obtenido un valor medio de 3,61 para el conjunto de los loci estudiados en la población global.

El coeficiente de consanguinidad estimado de forma indirecta a partir de las heterocigosidades observadas y esperad as de los 31 microsatélites de ADN analizados siguiend o la metodología propuesta por Nei y Kumar (2000), ha sido del 2,99 \%. Este valor (al igual que el de las propias heterocigosidades) muestra una situación de variabilidad genética lo suficientemente alta para que si se corrigen los problemas que se han iniciado (falta de rentabilidad, aislamiento geográfico y sanitario, envejecimiento de la población, dificultad de obtener machos reproductores, etc.) la raza no tenga problemas en superar la situación actual.

En los casos en que no existen registros genealógicos (como ocurre en la raza Pajuna) está cobrando protagonismo el llamado "parentesco molecular" basado en los marcadores genéticos como los microsatélites (Lynch and Ritland, 1999). Para la estimación de este parentesco molecular entre los diferentes individuos se ha utilizado el estimador R (Total Relatedness Estimate) definido por Wang (2002), a partir de los estimadores PHI y DELTA (Lynch and Ritland, 1999, Li et al., 1993). Este análisis se ha realizado tanto en la población global, como para cada una de las subpoblaciones analizadas mediante ADN (Jaén, Granada y Málaga). Según nuestros resultados en la población global se ha obtenido un parentesco medio de $4,54 \%$, con un $36,94 \%$ de los apareamientos con un parentesco superior a cero (media del 12,28\%).

Cuando realizamos este análisis bajo el supuesto de un flujo genético exclusivamente dentro de cada núcleo (el 46,82\% de los posibles apareamientos corresponden a parejas dentro del mismo núcleo geográfico), se observa que el parentesco medio ha sido lógicamente superior al de la población total (cruzamientos dentro y entre grupos geográficos) con una $\mathrm{R}$ promedio del $6,36 \% \mathrm{y}$ un $43,72 \%$ de los apareamientos consanguíneos (parentesco medio del $14,55 \%)$. En este caso el núcleo que posee un mayor coeficiente de parentesco molecular ha sido Granada ( $\mathrm{R}=8 \%)$ y el menor Málaga $(\mathrm{R}=1,35 \%)$. Así mismo han sido Granada y Málaga los núcleos que han presentado mayor y menor porcentaje de apareamientos consanguíneos con un $57,14 \%$ y el $3,00 \%$ respectivamente.

Cuando se estudian los apareamientos entre animales procedentes de distintos núcleos geográficos, el promedio para el parentesco molecular resulta sensiblemente menor (media de $2,93 \%$ ) que en el anterior apartado, lo que demostraría el escaso flujo que ha existido entre estos. Los núcleos más distantes genéticamente y que lógicamente presentan menor porcentaje de parentesco han sido Jaén x Málaga $(2,01 \%)$ y Málaga x Granada (2,13\%), coincidiendo con los resultados obtenidos en la estimación de las distancias genéticas calculadas a partir de los microsatélites de ADN. Así mismo, 
presentan los menores porcentajes de apareamientos consanguíneos con un $25 \%$ y $30 \%$ respectivamente.

\section{Medida de la variabilidad entre y dentro de núcleos de la raza Pajuna}

Se ha obtenido un valor medio de 0,026 para el $\mathrm{F}_{\text {IS }}$ (d efecto o exceso de heterocigotos en cada uno de los núcleos), 0,052 para $\mathrm{F}_{\text {IT }}$ (defecto o exceso de heterocigotos en la población global) y 0,027 para $\mathrm{F}_{\mathrm{ST}}$ (nivel de diferenciación genética). Este último valor indicaría la proporción de variación genética debida a la subdivisión de la población, es decir, muestra que casi el 3\% de la variabilidad genética total se debe a la diferencia existente entre los núcleos, el resto $(97 \%)$ sería debida a la diferencia entre individuos dentro de los grupos $(\mathrm{P}<0,05)$. Mediante la determinación del flujo de genes entre los 3 principales Núcleos de la raza mediante el número de migrantes por generación (Nm, Wright, 1969) se ha visto que ha sido en general bajo.

En la figura 4 se representa la distancia de Reynolds (Reynolds et al., 1983), una distancia que se ha mostrado muy adecuada para la diferenciación de poblaciones que han divergido hace muy poco tiempo. Según nuestros resultados la población bovina Pajuna está perfectamente diferenciada del resto de razas analizadas. Cuando este análisis se realiza incluyendo los diferentes núcleos de esta raza, el resultado muestra una baja distancia lo que indica que no se constituyen grupos bien diferenciados desde el punto de vista genético.

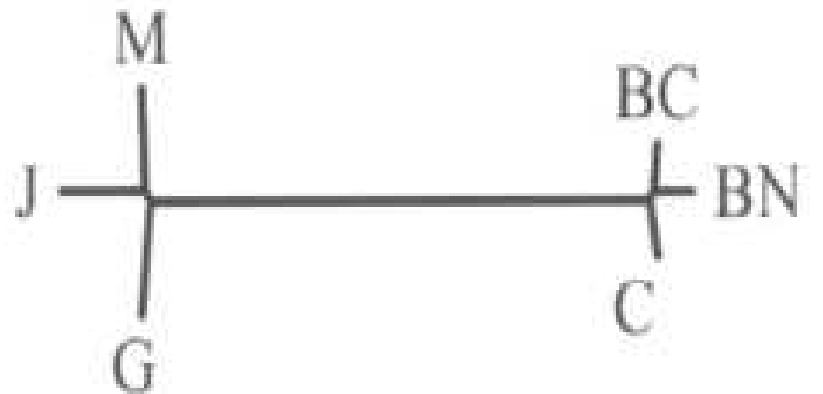

El análisis de asignación de los distintos individuos analizados (genotipos) a sus respectivas poblaciones ha mostrado una buena discriminación cuando se utilizan los 31 marcadores utilizados ya que en el peor de los casos (en el núcleo de Granada) contamos con una probabilidad del $71,4 \%$ de asignar correctamente un animal. En el núcleo de Jaén esta probabilidad es del $81,25 \%$ y en el caso de la población de Málaga del 100\%. La probabilidad global de asignar correctamente un individuo fue del $80,39 \%$. Tomando todos los núcleos de la raza Pajuna como una única población, la probabilidad de asignación de los animales a esta población ha sido del 98\%. Para el caso de la asignación de los individuos a las poblaciones de Berrenda en Colorado, Berrenda en Negro y Cárdena las probabilidades han sido del 92\% para las dos primeras y del $100 \%$ para la última. El verdadero valor de este estudio está en intentar determinar si el genotipado con el panel de microsatélites sería de utilidad para resolver aquellos casos en los que el historial del animal y/ o la etnología no son capaces de resolver la adscripción a una determinada raza (o el determinar el grado de pureza de un grupo de animales). En ningún caso pensamos que debe utilizarse para adscribir animales de los que se desconoce totalmente su historial y su morfología (Molina et al., 2003). La información proporcionada por marcadores moleculares neutros de tipo microsatélite describe similitudes entre poblaciones debidas a antecedentes comunes. Ponzoni (1997) establece que la información de marcadores moleculares neutros permite asegurar que poblaciones cuya apariencia y

\author{
G: núcleo de Granada \\ J: núcleo de Jaén \\ M: nú cleo de Málaga \\ BC, BN, C: Barrenda en Colorado \\ Barrenda en Negro y Cárdena, respectivamente
}

Figura 4. Árboles de distancias genéticas (Reynolds et al., 1983) entre los núcleos de la raza Pajuna y las otras razas. 
rendimientos son similares, son en realid ad diferentes, pero no que poblaciones cuya apariencia y rendimientos son diferentes sean genéticamente iguales.

Estimación de la variabilidad presente en la región control de la replicación del $\mathrm{ADN}$ mitocondrial

El análisis de las 53 secuencias obtenidas mostró 26 sitios polimórficos, configurando, a su vez, 26 haplotipos diferentes para el conjunto de individuos analizados (5 en la raza Pajuna). La raza Pajuna presenta claramente haplotipos de ADN mitocondrial de tipo europeo. Sin embargo, los haplotipos que presenta esta raza son mayoritariamente únicos (presenta 4 haplotipos singulares que no comparte con otras razas), lo que descartaría una introgresión directa vía hembra de las razas bovinas centroeuropeas estudiadas en las razas andaluzas y, especialmente, en la raza Pajuna. Más bien, los datos aportados en el presente análisis sugieren un origen ancestral común de la raza Pajuna respecto de las razas europeas (introgresión de ganado bovino que se llevó a cabo en Europa a partir de los lugares de domesticación del medio Oriente en tiempos prehistórico (Loftus et al.; 1994; Bradley et al., 1996; Troy et al., 2001) y un desarrollo autónomo respecto de ellas, si bien el grado de diferenciación entre el ganado europeo siempre debe entenderse como reducido.

Según nuestros resultados existiría una mayor lejanía entre la raza N'Dama y el resto de las razas (entre un 1,6 y un 2,1\%), mientras que entre las razas europeas es siempre inferior al $1 \%$ siendo la mayor entre las razas Limusín y Retinta $(0,9 \%)$ y la menor entre la raza Pajuna y la Charolesa $(0,3 \%)$. Por otra parte, la variabilidad intra raza presenta, en general, valores similares a las distancias entre razas, lo que caracteriza la existencia de una importante variabilidad dentro de cada población. Esto hace que la distancia media entre razas (dA), que tiene en cuenta la variabilidad intrapoblacional, sea cero para todas las parejas de razas europeas, mientras que la dA de la raza N'Dama respecto de cualquier otra raza europea ha sido del 1,1-1,2\%.

\section{Conclusiones}

Si atendemos exclusivamente al número de animales con cierto nivel de pureza la situación podría no ser considerada grave, pero si atendemos a los criterios de la EAAP (número de animales puros, la evolución censal, el porcentaje de cría en pureza etc.) está claro que la situación de esta raza es de extrema gravedad. No obstante, según nuestros resultados, esta raza ha mostrado un perfil genético definido y una entidad genética propia y un alto nivel de variabilidad genética. Ello permitirá su recuperación si se solucionan los problemas que la han llevado a la situación en la que se encuentra (aislamiento geográfico y sanitario, falta de rentabilidad de las explotaciones, aislamiento genético de los núcleos que la componen, etc.). En este sentido las medidas iniciadas por la Asociación de Criadores están siendo muy positivas para la recuperación y consolidación de la raza.

La especial importancia que tiene controlar la endogamia y el papel fundamental que tiene en el tamaño efectivo de la población el desequilibrio en el ratio semental/ vaca, nos obliga a que el plan de conservación que se establezca garantice que cada semental establezca un flujo de genes a los demás núcleos, y deje un número mínimo de descendientes en él, y que haya descendencia en cada núcleo de sementales de todos los núcleos.

Por último, las tres causas que hacemos responsables de la situación de la raza tienen un trasfondo social, la despoblación rural es un hecho, las actividades lúdicas tipo cinegética o turismo rural requieren menor esfuerzo para obtener mejores resultados, y otras actividades ganaderas más rentables, por lo que para invertir esta situación es necesario no sólo aumentar la renta de los ganaderos, sino profesionalizar sus sistemas 
de explotación con mejoras de las condiciones de trabajo y lograr la producción de un producto estandarizado y comercial, convirtiendo a los ganaderos en auténticos empresarios con visión de futuro, que animen a las nuevas generaciones a continuar con la actividad.

\section{Lista de Referencias}

Aparicio G. 1960. Zootecnia Especial. Etnología Compendiada. Imprenta moderna. Córdoba.

Azor P.J., A. Molina, M. Valera \& A. Luque. 2003. Diversidad genética de las subpoblaciones de ganado bovino Pajuno. VIII Jornadas Científicas de Veterinaria Militar. Madrid, España.

Bradley D.G., D.E. McHugh, E.P. Cunningham \& R.T. Loftus. 1996. Mitochondrial diversity and the origins of African and European cattle. Proc. Natl. Acad. Sci USA, 93, 5131-5135.

Cymbron T., R.T. Loftus, M.I. Malheiro \& D.G. Bradley. 1999. Mitochondrial sequence variation suggests an African influence in Portuguese cattle. Proc. R. Soc. Lond. B 266, 597-603.

El Mousadik A. \& R.J. Petit. 1996. High level of genetic differ-entiation for allelic richness among population of the argan tree [Argania spinosa (L.) Skeels] endemic to Morocco. Theor. Appl. Genet. 92, 832-839.

FAO. 1999. Secondary Guidelines for development of National Farm Animal Genetic Resources Management Plans. Measurement of Domestic animal Diversity (MoDAD): Recommended microsatellite markers.

Herrera M., F. Peña \& E. Rodero. 1996. Manual de campo para la identificación de las razas autóctonas en peligro de extinción en Andalucía. Ed. Junta de Andalucía, pp. 53.
Hill W. 1972 Effective size of populations with overlapping generations. Theor. Popul. Biol. 3, 278-289.

Hill W. 1979. A note on effective population size with overlapping generations. Genetics 92, 317-322.

Jordana J. \& O. Ribó. 1991. Relaciones filogenéticas entre razas ovinas españolas obtenidas a partir del estudio de caractres morfológicos. Investigaciones agrarias Producción y Saninad Animal. Vol 6. 3, 211-223.

Kimura M. \& J.F. Crows. 1963. The measurement of population number. Evolution. 17, 279-288.

Latter B. 1959. Genetic sampling in a random mating control population of constant size and sex ratio. Australian J. of Biol. Sci. 40, 500-505.

Li C.C., D.E. Weeks \& A. Chakravarti. 1993 Similarity of DNA fingerprints due to chance and relatedness. Hum. Hered. 43, 45-52.

Loftus R.T., D.E. MacHugh, D.G. Bradley, P.M. Sharp \& E.P. Cunningham. 1994. Evidence for two independent domestication of cattle. Proc. Natl. Acad. Sci USA, 91, 2757-2761.

Luque A., A. Molina \& Valera, M. 2001. Situación Actual de la raza bovina pajuna. Primeras Jornadas ibéricas de Razas Autóctonas y productos tradicionales. Elvas. Portugal.

Luque A., A. Molina, M. Valera, E. Rodero, A. Martínez \& P.J. Azor. 2002. La raza bovina Pajuna: Programa de Recuperación. V Congreso SERGA y III Congreso Ibérico sobre RGA, Madrid, España.

Luque A., A. Molina, M. Valera, P.J. Azor \& M. Luque. 2003. Caracterización del potencial para la producción de carne de la raza Pajuna. II Jornadas Ibéricas de razas autóctonas y sus productos tradicionales. Sevilla, España. 
Lynch M. \& K. Ritland. 1999. Estimation of pairwise relatedness with molecular markers. Genetics 152, 1753-1766.

Molina A., A. Luque, M. Valera, P.J. Azor, E. Rodero \& F. Goyache. 2003. Socioeconomic aspects of the Andalusian mountainous areas bovine of the Pajuna breed. EAAP International Mediterranean Symposium Animal Production and Natural Resources Utilization in Mediterranean Mountain Areas. Ioannina, Greece.

Molina A., A. Luque, M. Valera, P.J. Azor, E. Rodero \& F. Goyache. 2005. Socioeconomic aspects of the Andalusian mountainous areas bovine of the Pajuna breed. In: Animal production and natural resources utilisation in the Mediterranean mountain areas. EAAP Scientific Series no. 115. Ed. Wageningen Academic Publishers, pp. $432-435$.

Nei M. \& S. Kumar. 2000. Molecular Evolution and Phylogenetics. Oxford university press, pp. 236.

Paetkau D., W. Calvert, I. Stirling \& C. Strobeck. 1995. Microsatellite analysis of population structure in polar bears. Mol Ecol 4, 347-354.

Paetkau D., L.P. Waits, P.L. Clarkson, L. Craighead \& C. Strobeck. 1997. An empirical evaluation of genetic distance statistics using microsatellite data from bear (Ursidae) populations. Genetics 147, 1943-1957.

Ponzoni R.W. 1997. Genetic resources and conservation. In Piper and Ruvinsky (Eds), "The Genetics of Sheep", CAB International, Wallingford, pp. 437-469.

Reynolds J., B.S. Weir \& C.C. Cockerham. 1983. Estimation of the coancestry coefficient basis for a short-term genetic distance. Genetics 105, 767-779.

Rodero A. 1.999. Domestic Animal Diversity Information Siystem. www.fao.org/ dad-is, FAO, Rome, Italy.
Rodero E. 2003. Comunicación Personal.

Rodero E., M.E. Camacho, J.V. Delgado \& A. Rodero. 1992. Study of the Andalusian Minor breeds: Evaluation of the Priorities of conservation. Animal Genetic Resources Information 10, 41-52.

Rodero E., J.V. Delgado, A. Rodero \& M.E. Camacho. 1994. Conservación de razas autóctonas andaluzas en peligro de extinción. Ed. Consejería de Agricultura y Pesca. Junta de Andalucía. Sevilla, Spain.

Sánchez-Belda A. 1984. Razas Bovinas Españolas. Ed. Ministerio de Agricultura, Pesca y Alimentación.Madrid, Spain

Sánchez-Belda A. 2002. Razas Ganaderas Españolas Bovinas. Ed. Ministerio de Agricultura Pesca y Alimentación, Madrid, Spain, pp. 358.

Selander R.K. 1976. Genic Variation in Natural Populations. In: Molecular Evolution, Ayala F.D. (Ed.). Sunderland: Sinauer Associates, pp. 21-46.

Stoddart J.A. \& J.F. Taylor. 1985. Genotypic Diversity: Estimation and Prediction in Samples. Genetics, 118, 705-711.

Troy C.S., D.E. MacHugh, J.F. Bailey, D.A. Magee, R.T. Loftus, E.P. Cunningham, A.T. Chamberlain, B.C. Sykes \& D.G. Bradley. 2001. Genetic evidence for Near-Eastern origins of European cattle. Nature 410, 1088-1091.

Wang J. 2002. An Estimator for Pairwise Relatedness Using Molecular Markers. Genetics, 160, 1203-1215.

Wrigth S. 1931. Evolution Mendelian Populations. Genetics. 16, 107-111.

Wright S. 1969. Evolution and the genetics of Populations, Vol. 2. The theory of gene frequencies, University of Chicago, USA. 